\title{
Microwave tunable straight edge resonator on silicon membrane
}

\author{
George Sajin $^{(*)}$, Elena Matei ${ }^{(*)}$, Romolo Marcelli ${ }^{(* *)}$ \\ ${ }^{(*)}$ National Research and Development Institute for Microtechnologies, 32B Erou Iancu Nicolae \\ str., PO Box 38-160, 72996, Bucharest, Romania, Phone: +40-1-231.30.40, E-mail: gsajin@imt.ro; \\ elenama@imt.ro \\ ${ }^{(* *)} \mathrm{M}^{2} \mathrm{~T}$ - Microwave Microsystems Technology, CNR, Via del Fosso del Cavaliere 100, 00133 \\ Rome, Italy; E-mail: r.marcelli@psm.rm.cnr.it
}

\begin{abstract}
This work presents the experimental realization and the performances of a magnetostatic microwave straight edge tunable resonator (SER) placed on a silicon membrane. The aim of this approach is to study a miniaturised device, integrable in a planar micromachined microwave circuit. $S_{11}$ and $S_{21}$ parameters of this device are presented for different magnetic bias fields, exhibiting frequency tunability between $4.2 \mathrm{GHz}$ and 7.25 GHz. The utilization of silicon membranes to support these devices offers important openings toward the integration of magnetostatic wave devices with micromachined structures.
\end{abstract}

\section{INTRODUCTION}

The development of monolithic microwave integrated circuits imposes the necessity to assure the compatibility between the yttrium iron garnet (YIG) technology and the hybrid and monolithic configurations. Moreover, novel solutions for microwave and millimeter wave signal processing like those related to the realization of micromachined structures are suitable of an integration with magnetostatic wave devices. The configuration presented in this paper is a SER made by a YIG thin film, working in pure mode magnetostatic surface wave regime (MSSW), frequency tuned by changing the strength of an external bias magnetic field $\mathrm{H}_{\text {apl }}$. Electrically matched magnetostatic wave transducers have been realised by top-coupling the MSSW-SER to a dedicated micromachined microstrip structure designed and made in agreement with the characteristics of the etched silicon substrate.

\section{THEORETICAL BACKGROUND}

The resonator consists of a resonant cavity obtained by cutting a rectangular piece of a ferrimagnetic YIG film in the dimensions of few square millimeters from a "1" wafer YIG/GGG substrate obtained by liquid phase epitaxy. The edges of the SER are coupled with two transducers, a launcher and a detector, made by two grounded microstrip lines.

\section{A. Transducers on silicon membrane}

The microstrip transducers can theoretically include a number of $\mathrm{N}$ conducting strips, each of them having a width $a$ and carrying a current $\mathrm{I}_{0}$. They may form a microstrip line grid with $\mathrm{N}$ parallel conducting strips or a meander microstrip line with $\mathrm{N}$ meanders. The width $a$ of microstrip lines forming the launcher and detector structures have to be designed to have a $50 \quad \Omega$ characteristic impedance using relations in [1] and [2]. In our experiments $\mathrm{N}=1$. The $50 \mu \mathrm{m}$ thickness of the silicon membrane combined with a permitivity $\varepsilon_{\mathrm{r}}=11.7$ allows the design of microstrip lines $50 \mu \mathrm{m}$ wide (see also [3]).

\section{B. SER resonator structure}

For SER-MSSW, $\mathrm{H}_{\mathrm{apl}}$ lies in the plane of the ferrite thin film, normal on the direction of propagation. The magnetostatic waves induced 
by the launcher, reflected at the straight edges of the sample form a standing waves distribution that fulfils the relation (cf. [4]):

$$
\left(\mathrm{K}_{\mathrm{n}, \mathrm{m}}^{+}+\mathrm{K}_{\mathrm{n}, \mathrm{m}}^{-}\right) \times \mathrm{w}=2 \mathrm{n} \pi
$$

where $\mathrm{n}=1,2,3,(\mathrm{~K}+)$ and $(\mathrm{K}-)$ are the wavenumbers corresponding to the propagation at the YIG-GGG and at the YIGair interfaces respectively, and $w$ is the space between reflecting edges of the ferrite film. The subscript $m$ refers to the transversal resonant modes dependent on the width of the sample $w$ which may interfere with the main resonance.

One may demonstrate that at the YIG-GGG interface:

$$
\mathrm{K}_{\mathrm{n}, \mathrm{m}}{ }^{2}=\mathrm{K}_{\mathrm{m}}{ }^{2}+\left(\mathrm{m} \pi / 1 \omega_{\mathrm{h}}\right)^{2}
$$

and at the YIG-air interface:

$$
\mathrm{K}_{\mathrm{n}, \mathrm{m}}{ }^{2}=\mathrm{K}_{\mathrm{r}}^{2}+(\mathrm{m} \pi / 1)^{2}
$$

where

$$
\omega_{\mathrm{h}}=1-\frac{\omega \omega_{\mu}}{\omega^{2}-\omega_{\mu}^{2}}
$$

and

$$
\omega_{\mu}=\gamma \times 4 \pi \mathrm{M}_{0}
$$

For the design of the SER it is important to include the effects of both, material properties and geometry to properly account for intrinsic contributions and for boundary conditions effects. In particular, the material parameters are: the aturation magnetization $\left(4 \pi \mathrm{M}_{0}\right)$ that determines the minimum frequency obtainable with the resonator, the ferromagnetic resonance linewidth $(\Delta \mathrm{H})$ and the thickness of the epitaxial YIG layer $d$, while the geometrical parameters are: the length $l$ and the width $w$ (setting the magnetic wavelength) of the ferrite film, the distance $s$ between the microstrip transducers and the ferrite resonator (important for resonator coupling with launching and detecting microstrip lines), and the width of the transducers $a$ (important for reflection loss levels at the resonator input).

\section{DEVICE CONSTRUCTION AND EXPERIMENTAL RESULTS}

Using the above theoretical background a SER-MSSW resonator having a silicon membrane as substrate was designed and realized. The resonator structure on silicon membrane is shown in Fig.1.
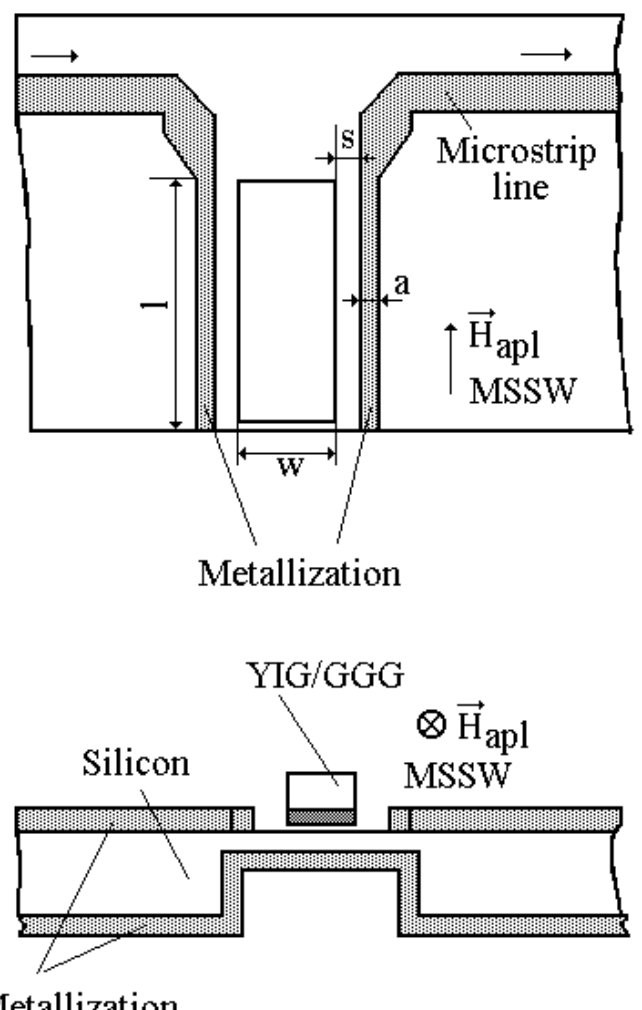

Metallization

Fig.1. The resonator structure on silicon membrane

To obtain the resonator structure, the following technological steps (cf. [5]) were performed:

- a <100> p-silicon wafer with a resistivity of $4000 \Omega . \mathrm{cm}$ was oxidised to obtain $1 \mu \mathrm{m}$ thick $\mathrm{SiO}_{2}$. This layer prevents the

silicon surface damage during the subsequent technological steps; 
- a $0.1 \mu \mathrm{m}$ SiNC layer was set down, this layer acting as a mask;

- the silicon was anisotropically etched to obtain a $50 \mu \mathrm{m}$ thick membrane;

- a metallisation with $50 \mathrm{~nm} \mathrm{Ti}$ (to ensure a better adhesion to the substrate) was followed by $1 \mu \mathrm{m}$ Au deposition (having in view the penetration depth up to $10 \mathrm{GHz}$ );

- a microstrip transducers pattern was obtained by a standard photolithographic process.

The transducers width/length dimmensions were $50 \mu \mathrm{m} / 4 \mathrm{~mm}$; the transition between transducer lines and feeding microstrip lines were designed to have a progressive impedance change to provide a large band operation for the SER.

The YIG material parameters were: $4 \pi \mathrm{M}_{0}=1750 \mathrm{Gs}, \Delta \mathrm{H}=4,1$ Oe and $\mathrm{d}=20 \mu \mathrm{m}$ on a $200 \mu \mathrm{m}$ thick GGG substrate. The optimal resonator dimensions, calculated from the above considerations in order to minimize the insertion losses, were: $1=4000 \mu \mathrm{m} ; \mathrm{w}=700$ $\mu \mathrm{m} ; \mathrm{s}=80 \mu \mathrm{m} ; \mathrm{a}=50 \mu \mathrm{m}$.

This device was characterized by measuring $S_{11}$ and $S_{21}$ for different $H_{a p l}$ values. Results are drawn in Fig.2. From these records one may see a tuning domain between $4.2 \mathrm{GHz}$ and 7.25 GHz. $\left|\mathrm{S}_{11}\right|$ values lie between $8 \mathrm{~dB}$ at $\mathrm{H}_{\mathrm{apl}}=540$ Oe and $16 \mathrm{~dB}$ at $\mathrm{H}_{\mathrm{apl}}=1980 \mathrm{Oe}$, which means a VSWR between 2.33 and 1.28. Losses, described by $\left|S_{21}\right|$, are $12 \mathrm{~dB}$ at $\mathrm{H}_{\mathrm{apl}}=540 \mathrm{Oe}$ and $7 \mathrm{~dB}$ at $\mathrm{H}_{\mathrm{apl}}=1980$ Oe.

\section{CONCLUSIONS}

In this paper it has been demonstrated the possibility to combine the planar YIG film technology with micromachining by studying a configuration made by a MSSW straight edge resonator excited by means of microstrip transducers on a silicon membrane. The measured tunability range of the device was between $4.2 \mathrm{GHz}$ and $7.25 \mathrm{GHz}$ and, in this frequency range, values of VSWR and insertion losses compatible with the data available in literature have been found. As a conclusion, a miniaturised magnetostatic wave device compatible with a micromachined structure has been obtained.

\section{REFERENCES}

[1] A.K. Ganguly, D.C. Webb "Microstrip excitation of magneto-static surface waves", IEEE Trans. on MTT, vol.23, p.998-1006, Dec.1975.

[2] Huijer E., Ishak W. "MSSW resonators with straight edge reflectors", IEEE Trans. on Mag. vol. 20(1984), no.5.

[3] Elena Matei, Sajin G. "S parameters for magnetostatic wave transducers on silicon microstructures", The Third International Conference on Low Dimensional Structures and Devices, LDSD'99, Antalya, Turkey, Sept. 15 - 17, 1999.

[4] Ishak W.S., et al. "Tunable microwave resonators using magnetostatic wave in YIG films", IEEE Trans. on MTT, vol. 34, no.12, Dec. 1986.

[5] Sajin G., Avramescu V., Dragoman M., Matei Elena, Marcelli R, "Magnetostatic wave transducers on silicon wafers and on silicon membranes", International Semiconductor Conference, CAS'98, Sinaia, Romania, p.635 - 638. 

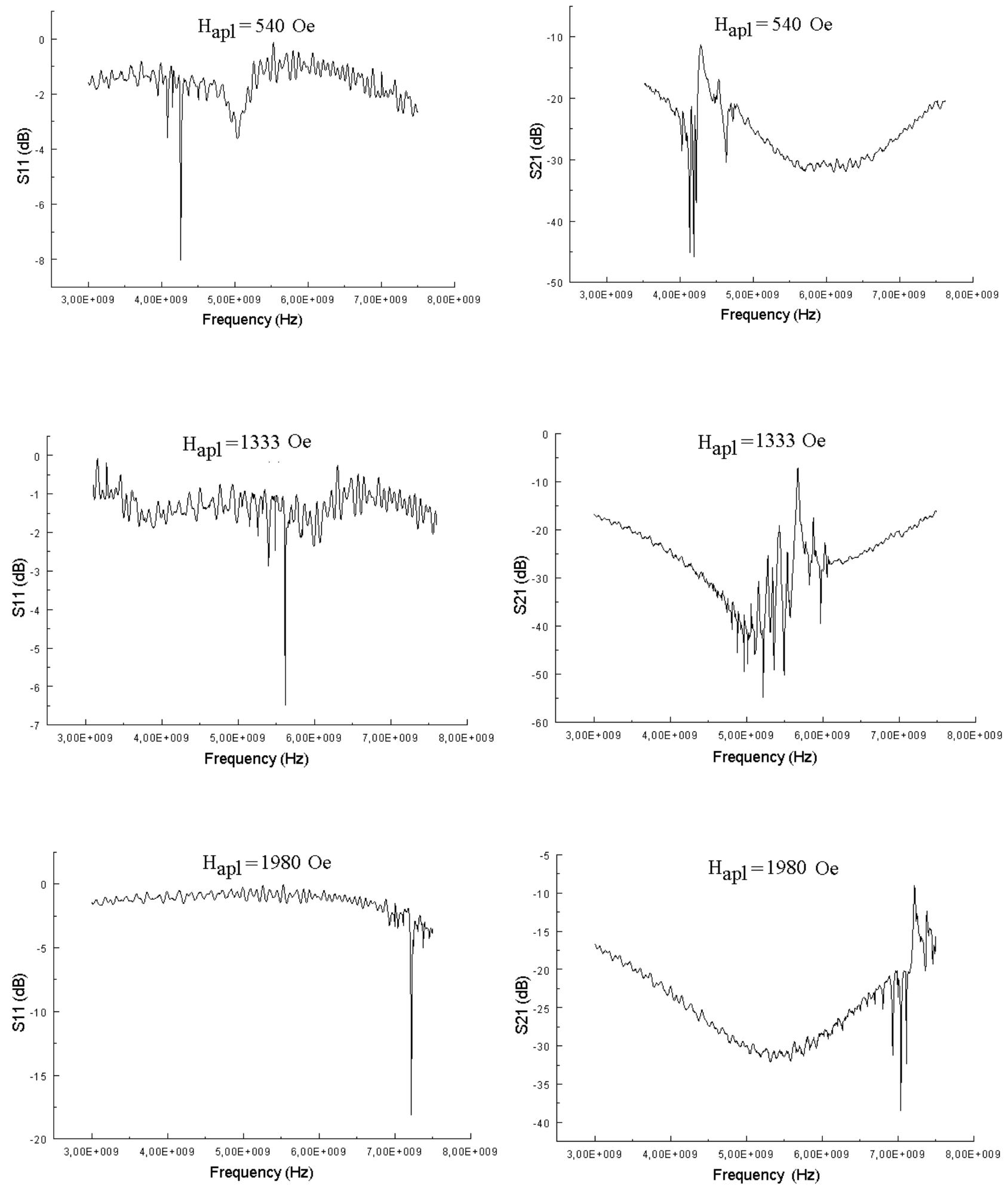

Fig. 2. SER $S_{11}$ and $S_{21}$ parameters for different applied magnetic field. 